Bull. Fac. Agric., Cairo Univ., 69:85-98(2018).

\title{
A STUDY ON VEGETATION DIVERSITY ALONG CAIRO - ISMAILIA DESERT ROAD, EGYPT
}

(Received: 14.1.2018)

\author{
By \\ S. A. Azer \\ Flora and Phytotaxonomy Researches Department, Horticultural Research Institute, Agricultural \\ Research Center, Giza, Egypt
}

\begin{abstract}
The aim of this research was to study vegetation diversity among the following habitats: field crops, orchards, gardens, roadsides, wastelands, salt marshes and deserts along Cairo - Ismailia desert road, Egypt. A total of 112 genera and 132 species belonging to 27 dicotyledons, 5 monocotyledons and 1 chlamydospermae families were recorded and identified with regional floras and available checklists. The common polytypic families were Poaceae 25 species followed by Compositae (21 species), then each of Brassicaceae and Chenopodiaceae was represented by 10 species. 15 families were monotypic. Grasses, subshrubs and sedges were represented by $21 \%, 3 \%$ and $2 \%$, respectively at polytypic families only. Annuals taxa presented the highest contributions $(65 \%)$ at polytypic families. while, biennials represented only $4 \%$ in monotypic families. The cluster analysis divided the studied habitats into five groups. Groups 1, 2 and 5 included the same habitats at monotypic and polytypic families, while, groups 2 and 3 contained different habitats in monotypic and polytypic ones. Field crops and orchards habitats showed the highest degree of similarity ratio $83.3 \%$ at monotypic and $60.7 \%$ in polytypic families. On the other hand, there was no degree of similarity ratios between species of salt marshes and the following habitats: field crops, orchards and gardens at monotypic families.
\end{abstract}

Key words: Vegetation, habit, life span, similarity, Cairo-Ismailia desert road.

\section{INTRODUCTION}

Desert vegetation is a characteristic feature in the inland part of the Eastern Desert where the ground water is shallow (Zahran and Willis, 2009 and Zahran and El-Amier, 2014). The Eastern Desert of Egypt occupies the area extending from the Nile Valley eastward to the Gulf of Suez and the Red Sea, which is about $223,000 \mathrm{~km}^{2}$, about $(22.3 \%)$ of the total area of Egypt. It is representing by numerous depression wadis running to the Red Sea or the Nile Valley. It consists of high rugged mountains that run parallel to a short distance from the coast (Salama et al., 2013). In the Eastern Desert, the natural vegetation occurs in the main stream of the wadis as well as on the slopes of the mountains that receive rainfall with mean annual up to $60 \mathrm{~mm}$ (Zahran and Willis, 2009 and Zahran and El-Amier, 2014). The plant life in the Eastern desert was studied botanically by different researchers (Kassas, 1953a, 1953b, 1954; Kassas and El-Abyad, 1962; Kassas and Girgis, 1964, 1965; Salama and Fayed, 1989,
1990; Salama and El-Naggar, 1991; Abd ElGhani, 1998; Boulos, 2008 and Salama, et al., 2013). Most of the previous studies dealt with the different ecological aspects, with less attention to the floristic features of this desert. The Cairo - Ismailia desert road is located in the northern part of the Eastern desert and extends from Cairo till Ismailia cities (about 128km long). The aim of this study was to provide a description of the vegetation diversity among the studied habitats: field crops, orchards, gardens, roadsides, wastelands, salt marshes and deserts along Cairo - Ismailia desert road.

\section{MATERIALS AND METHODS}

The study area extended from Cairo till Ismailia cities, about $128 \mathrm{~km}$ long, and located between $\left(30^{\circ} 05^{\prime} 04^{\prime \prime} \mathrm{N}-3035^{\circ} 09^{\prime \prime} \mathrm{N}\right.$ and $31^{\circ}$ 14' 30" E- 32० 15' 49" E) (Fig. 1). Several visits were performed along Cairo-Ismailia desert road from March 2016 till March 2017. Field data were gathered from the following habitats: field crops, orchards, gardens, roadsides, wastelands, 


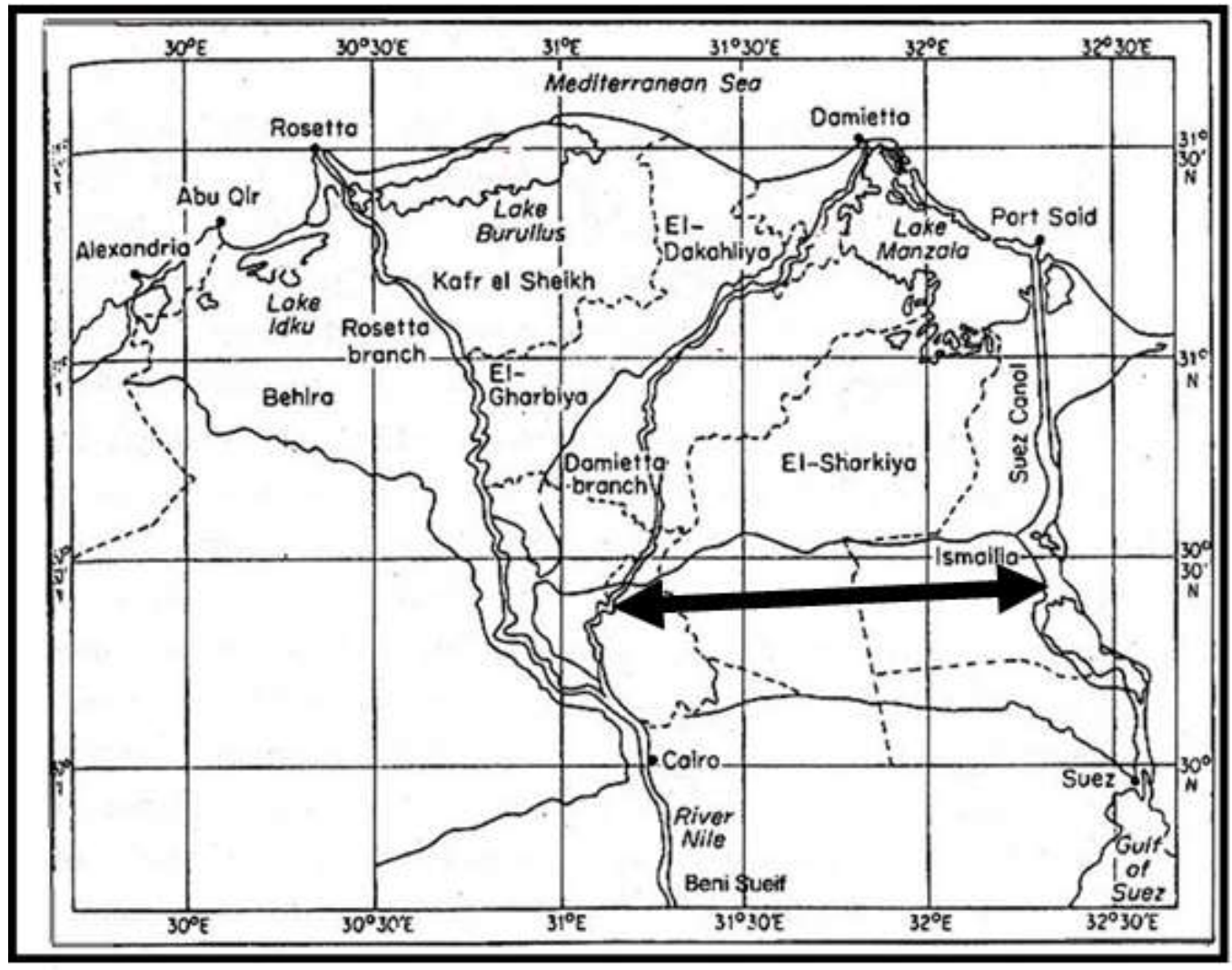

Fig. (1): A map of the location of Cairo - Ismailia desert road, Egypt.

salt marshes and deserts. The collected species were recorded to represent the vegetation diversity among the studied habitats. The recorded species were arranged alphabetically with their families and genera. Voucher specimens were collected and identified at the herbarium of Flora and Phytotaxonomy Research Department (CAIM), Horticultural Research Institute, Agricultural Research Center, Giza, Egypt and arranged alphabetically with their families. Species identification was done according to Täckholm (1974); El-Hadidi, and Fayed (1994/95); Boulos (1995, 1999, 2000, 2002, 2005, 2009) and updated by Angiosperm Phylogeny Group III (2009); Chase and Reveal (2009) and Haston et al., (2009). Numerical analysis of the studied habitats was carried out and based on hierarchical cluster analysis. The retrieved output was used to construct specific ecological relationships among the studied habitats. The data were treated as a binary character using SPSS version 22 (SPSS, 2013). The output was plotted in the form of dendrogram. The dendrogram was based on average linkage (between groups) and rescaled distance cluster combine.

\section{RESULTS AND DISCUSSION}

\subsection{Floristic composition of monotypic and} polytypic families

The lists of species were arranged alphabetically with their monotypic and polytypic families and genera. The presence or absence values among the studied habitats are listed in (Tables $1 \& 2$ ). The current study recorded the presence of 132 species, representing 112 genera (Table 3). The most distributed polytypic families were Poaceae and Compositae. Poaceae was represented by 25 species (18.94\%) and Compositae by 21 species (15.91\%). In addition, both of Brassicaceae and Chenopodiaceae were 10 species $(7.58 \%)$. In addition, the following families: Zygophyllaceae and Euphorbiaceae were represented by 5 species (3.79\%). Moreover, 4 species were recorded from each of Aizoaceae, Amaranthaceae, Convolvulaceae and Solanaceae and 3 species from each of Apiaceae, Malvaceae and Polygonaceae. On the other hand, 15 families were monotypic. The four major distributed families (Poaceae, Compositae, Brassicaceae and Chenopodiaceae) were reported earlier by Mashaly et al. (2009); Hamed et al. (2012); Azer (2013) and Amer et al.(2015) 
Table (1): List of monotypic families and data matrix of 15 species distributed among the studied habitats along Cairo-Ismailia desert road.

\begin{tabular}{|c|c|c|c|c|c|c|c|c|c|c|c|}
\hline \multirow[b]{2}{*}{ No. } & \multirow[b]{2}{*}{ Families and taxa } & \multicolumn{3}{|c|}{ Vegetation traits } & \multicolumn{7}{|c|}{ Studied habitats } \\
\hline & & "a & 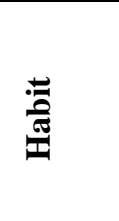 & 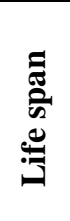 & 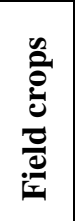 & 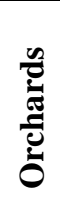 & 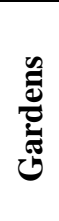 & 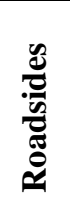 & 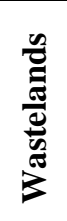 & 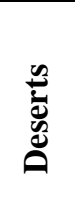 & 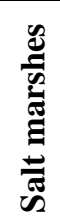 \\
\hline & Arecaceae & $\mathbf{M}$ & & & & & & & & & \\
\hline \multirow[t]{2}{*}{1} & Phoenix dactylifera $\mathrm{L}$. & & tree & $\mathrm{P}$ & 1 & 1 & 1 & 1 & 1 & 1 & 0 \\
\hline & Boraginaceae & D & & & & & & & & & \\
\hline \multirow[t]{2}{*}{2} & Heliotropium arbainense Fresen. & & herb & $\mathrm{P}$ & 0 & 1 & 0 & 0 & 0 & 1 & 0 \\
\hline & Cleomaceae & D & & & & & & & & & \\
\hline \multirow[t]{2}{*}{3} & Cleome droserifolia (Forssk.) Delile & & herb & $\mathrm{P}$ & 0 & 0 & 0 & 0 & 0 & 1 & 0 \\
\hline & Cucurbitaceae & D & & & & & & & & & \\
\hline \multirow[t]{2}{*}{4} & Citrullus colocynthis (L.) Schrad. & & herb & $\mathrm{P}$ & 0 & 0 & 0 & 0 & 0 & 1 & 0 \\
\hline & Ephedraceae & C & & & & & & & & & \\
\hline \multirow[t]{2}{*}{5} & Ephedra alata Decne. & & shrub & $\mathrm{P}$ & 0 & 0 & 0 & 0 & 0 & 1 & 0 \\
\hline & Geraniaceae & D & & & & & & & & & \\
\hline \multirow[t]{2}{*}{6} & Erodium laciniatum (Cav.) Willd. & & herb & A & 0 & 0 & 0 & 0 & 0 & 1 & 0 \\
\hline & Juncaceae & M & & & & & & & & & \\
\hline \multirow[t]{2}{*}{7} & Juncus bufonius L. & & rush & A & 0 & 0 & 0 & 0 & 0 & 0 & 1 \\
\hline & Neuradaceae & D & & & & & & & & & \\
\hline \multirow[t]{2}{*}{8} & Neurada procumbens L. & & herb & A & 0 & 0 & 0 & 0 & 0 & 1 & 0 \\
\hline & Oxalidaceae & D & & & & & & & & & \\
\hline \multirow[t]{2}{*}{9} & Oxalis corniculata $\mathrm{L}$. & & herb & $\mathrm{P}$ & 1 & 1 & 0 & 0 & 0 & 0 & 0 \\
\hline & Portulacaceae & D & & & & & & & & & \\
\hline \multirow[t]{2}{*}{10} & Portulaca oleracea L. & & herb & $\mathrm{A}$ & 1 & 1 & 1 & 1 & 0 & 0 & 0 \\
\hline & Primulaceae & D & & & & & & & & & \\
\hline \multirow[t]{2}{*}{11} & Anagallis arvensis $\mathrm{L}$. & & herb & $\mathrm{A}$ & 1 & 1 & 1 & 0 & 0 & 0 & 0 \\
\hline & Resedaceae & D & & & & & & & & & \\
\hline \multirow[t]{2}{*}{12} & Ochradenus baccatus Delile & & shrub & $\mathrm{P}$ & 0 & 0 & 0 & 0 & 0 & 1 & 0 \\
\hline & Tamaricaceae & $\mathbf{D}$ & & & & & & & & & \\
\hline \multirow[t]{2}{*}{13} & Tamarix senegalensis DC. & & shrub & $\mathrm{P}$ & 0 & 0 & 0 & 1 & 1 & 1 & 1 \\
\hline & Typhaceae & $\mathbf{M}$ & & & & & & & & & \\
\hline \multirow[t]{2}{*}{14} & Typha domingensis Pers. & & herb & $\mathrm{P}$ & 0 & 0 & 0 & 0 & 0 & 0 & 1 \\
\hline & Urticaceae & D & & & & & & & & & \\
\hline 15 & Urtica urens L. & & herb & A & 1 & 1 & 0 & 0 & 0 & 0 & 0 \\
\hline
\end{tabular}

Division: $(\mathbf{C}=$ Chlamydospermae, $\mathbf{D}=$ Dicotyledons, $\mathbf{M}=$ Monocotyledons $)$. Life span: $(\mathbf{A}=$ Annuals, $\mathbf{P}=$ Perennials $)$. Data matrix: $(1=$ presence, $0=$ absence $)$.

as the most frequent families in the studied areas. Similar conclusion has been reached by Shaheen (2002) and Abd El-Ghani and Fawzy (2006).

\subsection{Degree of similarity between monotypic and polytypic families}

The data presented in (Table 4), showed that field crops and orchard habitats showed the highest degree of similarity ratio $83.3 \%$ followed by $66.7 \%$ between roadsides and wastelands habitats, while the least ratio $7.7 \%$ was recorded between field crops and deserts habitat at the monotypic families. On the other hand, there was no degree of similarity value between salt marshes habitat and the following ones: field crops, orchards and gardens. Regarding, the polytypic families (Table 5) the highest degree of similarity ratio $60.7 \%$ was recorded between field crops and orchards habitats, followed by $46.9 \%$ between gardens and roadsides habitats. 
Table (2): List of polytypic families and data matrix of 117 species distributed among the studied habitats along Cairo-Ismailia desert road.

\begin{tabular}{|c|c|c|c|c|c|c|c|c|c|c|c|}
\hline \multirow[b]{2}{*}{ No. } & \multirow[b]{2}{*}{ Families and taxa } & \multicolumn{3}{|c|}{ Vegetation traits } & \multicolumn{7}{|c|}{ Studied habitats } \\
\hline & & 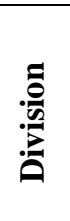 & 誩 & 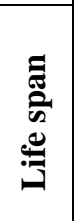 & 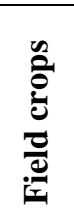 & 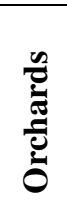 & 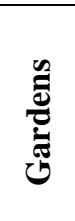 & 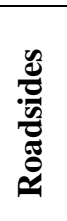 & 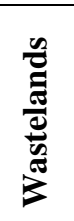 & 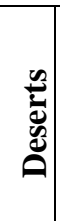 & 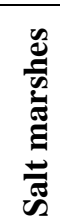 \\
\hline & Aizoaceae & D & & & & & & & & & \\
\hline 1 & Aizoon canariense $\mathrm{L}$. & & herb & A & 0 & 0 & 0 & 1 & 0 & 1 & 0 \\
\hline 2 & Mesembryanthemum crystallinum L. & & herb & A & 0 & 0 & 0 & 1 & 0 & 1 & 0 \\
\hline 3 & Mesembryanthemum forskahlii Hochst. ex Boiss. & & herb & A & 0 & 0 & 0 & 1 & 0 & 1 & 0 \\
\hline \multirow[t]{2}{*}{4} & Trianthema portulacastrum $\mathrm{L}$. & & herb & A & 0 & 0 & 1 & 1 & 1 & 0 & 0 \\
\hline & Amaranthaceae & D & & & & & & & & & \\
\hline 5 & Aerva javanica (Burm.f.) Juss. ex Schult. & & subshrub & $\mathrm{P}$ & 0 & 0 & 0 & 0 & 0 & 1 & 0 \\
\hline 6 & Amaranthus blitum subsp. oleraceus (L.) Costea & & herb & $\mathrm{A}$ & 1 & 1 & 1 & 1 & 0 & 0 & 0 \\
\hline 7 & Amaranthus hybridus L. & & herb & A & 1 & 0 & 1 & 0 & 0 & 0 & 0 \\
\hline \multirow[t]{2}{*}{8} & Amaranthus viridis $\mathrm{L}$. & & herb & A & 1 & 0 & 1 & 0 & 0 & 0 & 0 \\
\hline & Apiaceae & D & & & & & & & & & \\
\hline 9 & Ammi majus $\mathrm{L}$. & & herb & A & 1 & 0 & 1 & 0 & 0 & 0 & 0 \\
\hline 10 & Ammi visnaga $(\mathrm{L}$.) Lam. & & herb & A & 1 & 0 & 1 & 0 & 0 & 0 & 0 \\
\hline \multirow[t]{2}{*}{11} & Deverra tortuosa (Desf.) DC. & & herb & $\mathrm{P}$ & 0 & 0 & 0 & 0 & 0 & 1 & 0 \\
\hline & Apocynaceae & D & & & & & & & & & \\
\hline 12 & Calotropis procera (Aiton) Dryand. & & shrub & $\mathrm{P}$ & 0 & 0 & 0 & 1 & 1 & 1 & 0 \\
\hline \multirow[t]{2}{*}{13} & Cynanchum acutum $\mathrm{L}$. & & herb & $\mathrm{P}$ & 0 & 1 & 1 & 1 & 1 & 0 & 0 \\
\hline & Brassicaceae & $\mathbf{D}$ & & & & & & & & & \\
\hline 14 & Brassica nigra (L.) K. Kotch & & herb & A & 1 & 1 & 0 & 1 & 0 & 0 & 0 \\
\hline 15 & Brassica tournefortii Gouan & & herb & $\mathrm{A}$ & 1 & 1 & 0 & 1 & 0 & 0 & 0 \\
\hline 16 & Capsella bursa-pastoris (L.) Medik. & & herb & $\mathrm{A}$ & 1 & 1 & 0 & 0 & 0 & 0 & 0 \\
\hline 17 & Eruca vesicaria (L.) Cav. & & herb & A & 1 & 1 & 1 & 1 & 0 & 0 & 0 \\
\hline 18 & Farsetia aegyptia Turra. & & herb & $\mathrm{P}$ & 0 & 0 & 0 & 0 & 0 & 1 & 0 \\
\hline 19 & Matthiola longipetala (Vent.) DC. & & herb & A & 0 & 0 & 0 & 1 & 0 & 1 & 0 \\
\hline 20 & Raphanus raphanistrum $\mathrm{L}$. & & herb & $\mathrm{A}$ & 1 & 1 & 0 & 0 & 0 & 0 & 0 \\
\hline 21 & Sinapis alba $\mathrm{L}$. & & herb & $\mathrm{A}$ & 0 & 0 & 1 & 1 & 0 & 0 & 0 \\
\hline 22 & Sisymbrium irio L. & & herb & $\mathrm{A}$ & 1 & 1 & 1 & 1 & 0 & 0 & 0 \\
\hline \multirow{2}{*}{23} & Zilla spinosa (L.) Prantl & & shrub & $\mathrm{P}$ & 0 & 1 & 0 & 0 & 0 & 1 & 0 \\
\hline & Caryophyllaceae & D & & & & & & & & & \\
\hline 24 & Gypsophila capillaris (Forssk.) C. Chr. & & herb & $\mathrm{P}$ & 0 & 0 & 0 & 0 & 0 & 1 & 0 \\
\hline \multirow[t]{2}{*}{25} & Herniaria hirsuta L. & & herb & A & 0 & 0 & 0 & 0 & 0 & 1 & 0 \\
\hline & Chenopodiaceae & D & & & & & & & & & \\
\hline 26 & Anabasis articulata (Forssk.) Moq. & & shrub & $\mathrm{S}$ & 0 & 0 & 0 & 0 & 0 & 1 & 0 \\
\hline 27 & Anabasis setifera Moq. & & subshrub & $\mathrm{S}$ & 0 & 0 & 0 & 0 & 0 & 1 & 0 \\
\hline 28 & Atriplex prostrata subsp. calotheca $(\mathrm{Rafn})$ M.A.Gust. & & herb & A & 0 & 1 & 0 & 0 & 0 & 1 & 1 \\
\hline 29 & Bassia indica (Wight) A.J.Scott & & herb & A & 0 & 0 & 1 & 1 & 1 & 1 & 0 \\
\hline 30 & Bassia muricata (L.) Asch. & & herb & A & 0 & 1 & 0 & 0 & 1 & 1 & 0 \\
\hline 31 & Beta vulgaris $\mathrm{L}$. & & herb & $\mathrm{A}$ & 1 & 1 & 0 & 0 & 0 & 0 & 0 \\
\hline 32 & Chenopodium album $\mathrm{L}$. & & herb & $\mathrm{A}$ & 1 & 1 & 1 & 1 & 1 & 0 & 0 \\
\hline 33 & Chenopodium murale $\mathrm{L}$. & & herb & $\mathrm{A}$ & 1 & 1 & 1 & 1 & 1 & 0 & 0 \\
\hline 34 & $\begin{array}{l}\text { Chenopodium ambrosioides (L.) Mosyakin \& } \\
\text { Clemants }\end{array}$ & & herb & $\mathrm{B}$ & 1 & 1 & 1 & 1 & 0 & 0 & 0 \\
\hline \multirow[t]{2}{*}{35} & Haloxylon salicornicum (Moq.) Bunge ex Boiss. & & herb & $\mathrm{P}$ & 0 & 0 & 0 & 0 & 0 & 1 & 0 \\
\hline & Compositae & D & & & & & & & & & \\
\hline 36 & Achillea fragrantissima (Forssk.) Sch. Bip. & & herb & $\mathrm{P}$ & 0 & 0 & 0 & 0 & 0 & 1 & 0 \\
\hline
\end{tabular}


Table (2): Continued I

\begin{tabular}{|c|c|c|c|c|c|c|c|c|c|c|c|}
\hline & & Veg & tation tra & & Stu & lied $\mathrm{l}$ & abita & & & & \\
\hline No. & Families and taxa & 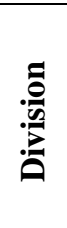 & 言 & 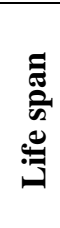 & 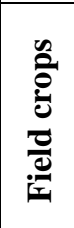 & 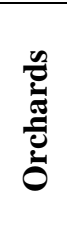 & שֶ: & 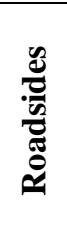 & 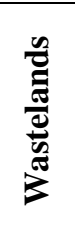 & 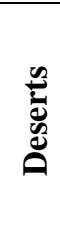 & 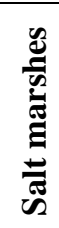 \\
\hline 37 & Artemisia monosperma Delile & & subshrub & $\mathrm{P}$ & 0 & 0 & 0 & 0 & 0 & 1 & 0 \\
\hline 38 & Bidens pilosa $\mathrm{L}$. & & herb & A & 0 & 1 & 1 & 0 & 0 & 0 & 0 \\
\hline 39 & Brocchia cinerea (Delile) Vis. & & herb & A & & & & & & & \\
\hline 40 & Centaurea aegyptiaca $\mathrm{L}$. & & herb & B & 0 & 0 & 0 & 0 & 0 & 1 & 0 \\
\hline 41 & $\begin{array}{l}\begin{array}{l}\text { Cichorium endivia } \\
\text { (Schousb.) P.D. Sell }\end{array} \\
\text { L. subsp. divaricatum } \\
\end{array}$ & & herb & A & 1 & 0 & 0 & 0 & 1 & 0 & 0 \\
\hline 42 & Echinops spinosissimus Turra & & herb & $\mathrm{P}$ & 0 & 0 & 0 & 0 & 0 & 1 & 0 \\
\hline 43 & Eclipta prostrata $(\mathrm{L}.) \mathrm{L}$. & & herb & A & 1 & 0 & 0 & 0 & 0 & 0 & 0 \\
\hline 44 & Erigeron bonariensis $\mathrm{L}$. & & herb & A & 1 & 1 & 0 & 0 & 0 & 0 & 0 \\
\hline 45 & Iphiona mucronata (Forssk.) Asch. \& Schweinf. & & herb & $\mathrm{P}$ & 0 & 0 & 0 & 0 & 0 & 1 & 0 \\
\hline 46 & Lactuca serriola $\mathrm{L}$. & & herb & $\mathrm{B}$ & 0 & 0 & 0 & 0 & 0 & 1 & 0 \\
\hline 47 & Laphangium luteoalbum (L.) Tzvelev & & herb & A & 0 & 0 & 1 & 1 & 1 & 0 & 0 \\
\hline 48 & Launaea mucronata (Forssk.) Muschl. & & herb & B & 0 & 0 & 0 & 0 & 0 & 1 & 0 \\
\hline 49 & Launaea nudicaulis (L.) Hook.f. & & herb & $\mathrm{P}$ & 0 & 1 & 1 & 1 & 0 & 1 & 0 \\
\hline 50 & Matricaria chamomilla $\mathrm{L}$. & & herb & A & 0 & 0 & 1 & 1 & 0 & 0 & 0 \\
\hline 51 & Pluchea dioscoridis (L.) DC. & & herb & $\mathrm{P}$ & 0 & 0 & 0 & 0 & 1 & 1 & 1 \\
\hline 52 & Pulicaria undulata (L.) C.A.Mey. & & herb & $\mathrm{P}$ & 0 & 0 & 0 & 0 & 0 & 1 & 0 \\
\hline 53 & Reichardia tingitana $(\mathrm{L}$.$) Roth$ & & herb & A & 0 & 1 & 0 & 0 & 1 & 1 & 0 \\
\hline 54 & Senecio desfontainei Druce & & herb & A & 0 & 1 & 1 & 1 & 0 & 1 & 0 \\
\hline 55 & $\begin{array}{l}\text { Sericocarpus linifolius (L.) "Britton, Sterns \& } \\
\text { Poggenb. }\end{array}$ & & herb & $\mathrm{A}$ & 0 & 0 & 1 & 0 & 1 & 0 & 0 \\
\hline 56 & Sonchus oleraceus $(\mathrm{L}.) \mathrm{L}$. & & herb & A & 1 & 1 & 1 & 1 & 1 & 0 & 0 \\
\hline & Convolvulaceae & & & & & & & & & & \\
\hline 57 & Convolvulus arvensis L. & D & herb & $\mathrm{P}$ & 1 & 1 & 1 & 1 & 1 & 0 & 0 \\
\hline 58 & Convolvulus lanatus Vahl & & herb & $\mathrm{P}$ & 0 & 0 & 0 & 0 & 0 & 1 & 0 \\
\hline 59 & Ipomoea cairica (L.) Sweet & & herb & $\mathrm{P}$ & 0 & 0 & 0 & 0 & 1 & 0 & 0 \\
\hline 60 & Ipomoea carnea Jacq. & & shrub & $\mathrm{P}$ & 0 & 0 & 0 & 0 & 1 & 0 & 0 \\
\hline & Cyperaceae & $\mathbf{M}$ & & & & & & & & & \\
\hline 61 & Cyperus articulatus $\mathrm{L}$. & & sedge & $\mathrm{P}$ & 0 & 0 & 0 & 0 & 0 & 0 & 1 \\
\hline 62 & Cyperus rotundus $\mathrm{L}$. & & sedge & $\mathrm{P}$ & 1 & 1 & 1 & 1 & 1 & 0 & 0 \\
\hline & Euphorbiaceae & D & & & & & & & & & \\
\hline 63 & Chrozophora tinctoria (L.) A.Juss. & & herb & A & 0 & 0 & 0 & 0 & 0 & 1 & 0 \\
\hline 64 & Euphorbia heterophylla $\mathrm{L}$. & & herb & A & 0 & 0 & 1 & 1 & 1 & 0 & 0 \\
\hline 65 & Euphorbia peplus L. & & herb & A & 1 & 1 & 1 & 1 & 0 & 0 & 0 \\
\hline 66 & Euphorbia retusa Forssk. & & herb & A & 0 & 0 & 0 & 0 & 0 & 1 & 0 \\
\hline 67 & Ricinus communis L. & & shrub & $\mathrm{S}$ & 1 & 1 & 0 & 0 & 1 & 0 & 0 \\
\hline & Leguminosae & D & & & & & & & & & \\
\hline 68 & Alhagi graecorum Boiss. & & herb & $\mathrm{P}$ & 0 & 1 & 0 & 1 & 1 & 0 & 0 \\
\hline 69 & Leucaena leucocephala (Lam.) De Wit & & tree & $\mathrm{T}$ & 0 & 0 & 1 & 0 & 1 & 0 & 0 \\
\hline 70 & Lotus glaber Mill. & & herb & A & 0 & 0 & 0 & 1 & 0 & 0 & 0 \\
\hline 71 & Medicago polymorpha $\mathrm{L}$. & & herb & $\mathrm{A}$ & 1 & 1 & 1 & 0 & 0 & 0 & 0 \\
\hline 72 & Melilotus indicus (L.) All. & & herb & A & 1 & 1 & 1 & 0 & 0 & 0 & 0 \\
\hline 73 & Sesbania sesban (L.) Merr. & & shrub & $\mathrm{S}$ & 1 & 1 & 0 & 0 & 1 & 0 & 0 \\
\hline 74 & Trifolium alexandrinum $\mathrm{L}$. & & herb & $\mathrm{A}$ & 1 & 1 & 1 & 0 & 1 & 0 & 0 \\
\hline 75 & Trifolium resupinatum L. & & herb & $\mathrm{A}$ & 1 & 1 & 0 & 0 & 1 & 0 & 0 \\
\hline & Malvaceae & D & & & & & & & & & \\
\hline 76 & Corchorus olitorius L. & & herb & A & 1 & 1 & 0 & 0 & 1 & 0 & 0 \\
\hline 77 & Malva parviflora $\mathrm{L}$. & & herb & $\mathrm{A}$ & 1 & 1 & 1 & 1 & 1 & 1 & 0 \\
\hline
\end{tabular}


Table (2): Continued II

\begin{tabular}{|c|c|c|c|c|c|c|c|c|c|c|c|}
\hline \multirow[b]{2}{*}{ No. } & \multirow[b]{2}{*}{ Families and Taxa } & \multicolumn{3}{|c|}{ Vegetation traits } & \multicolumn{7}{|c|}{ Studied habitats } \\
\hline & & 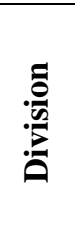 & 蒿 & 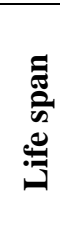 & 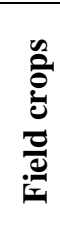 & 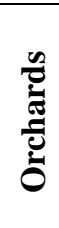 & 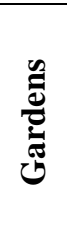 & 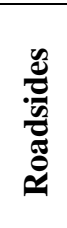 & 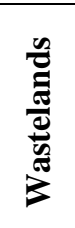 & 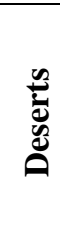 & 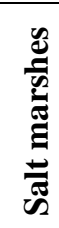 \\
\hline 78 & Sida spinosa $\mathrm{L}$. & & herb & $\mathrm{P}$ & 0 & 0 & 0 & 0 & 1 & 0 & 0 \\
\hline & Plantaginaceae & D & & & & & & & & & \\
\hline 79 & Plantago ovata Forssk. & & herb & A & 0 & 1 & 1 & 0 & 0 & 1 & 0 \\
\hline 80 & Plantago major $\mathrm{L}$. & & herb & A & 1 & 1 & 1 & 1 & 0 & 0 & 0 \\
\hline & Poaceae & $\mathbf{M}$ & & & & & & & & & \\
\hline 81 & Aristida mutabilis Trin. \& Rupr. & & grass & A & 0 & 0 & 0 & 0 & 0 & 1 & 0 \\
\hline 82 & Avena fatua $\mathrm{L}$. & & grass & A & 1 & 0 & 1 & 0 & 0 & 0 & 0 \\
\hline 83 & Avena sativa $\mathrm{L}$. & & grass & A & 1 & 0 & 1 & 0 & 0 & 0 & 0 \\
\hline 84 & Cenchrus biflorus Roxb. & & grass & A & 0 & 1 & 1 & 1 & 0 & 0 & 0 \\
\hline 85 & Cynodon dactylon (L.) Pers. & & grass & $\mathrm{P}$ & 1 & 1 & 1 & 1 & 1 & 1 & 1 \\
\hline 86 & Desmostachya bipinnata (L.) Stapf. & & grass & A & 0 & 1 & 0 & 0 & 1 & 0 & 0 \\
\hline 87 & Digitaria sanguinalis $(\mathrm{L}$.$) Scop.$ & & grass & A & 0 & 1 & 1 & 0 & 0 & 0 & 0 \\
\hline 88 & Echinochloa colona (L.) Link. & & grass & A & 1 & 1 & 0 & 1 & 0 & 0 & 0 \\
\hline 89 & Echinochloa stagnina (Retz.) P. Beauv. & & grass & A & 1 & 0 & 0 & 1 & 0 & 0 & 0 \\
\hline 90 & $\begin{array}{l}\text { Hordeum murinum L. subsp. leporinum (Link) } \\
\text { Arcang. }\end{array}$ & & grass & $\mathrm{A}$ & 0 & 0 & 1 & 1 & 0 & 1 & 0 \\
\hline 91 & Imperata cylindrica $($ L.) Raeusch. & & grass & $\mathrm{P}$ & 0 & 0 & 0 & 0 & 1 & 0 & 1 \\
\hline 92 & Lolium multiflorum Lam. & & grass & A & 1 & 1 & 1 & 1 & 0 & 0 & 0 \\
\hline 93 & Panicum coloratum L. & & grass & A & 1 & 1 & 1 & 0 & 0 & 0 & 0 \\
\hline 94 & Panicum turgidum Forssk. & & grass & $\mathrm{P}$ & 0 & 0 & 0 & 0 & 0 & 1 & 0 \\
\hline 95 & $\begin{array}{l}\text { Pennisetum divisum (Forssk. ex J.F.Gmel.) } \\
\text { Henrard }\end{array}$ & & grass & $\mathrm{P}$ & 0 & 0 & 0 & 0 & 0 & 1 & 0 \\
\hline 96 & Phalaris minor Retz. & & grass & A & 1 & 1 & 1 & 1 & 0 & 0 & 0 \\
\hline 97 & Phragmites australis (Cav.) Trin. ex Steud. & & grass & $\mathrm{P}$ & 0 & 1 & 0 & 0 & 1 & 0 & 1 \\
\hline 98 & Роа аппиа L. & & grass & A & 1 & 1 & 1 & 0 & 0 & 0 & 0 \\
\hline 99 & Polypogon monspeliensis (L.) Desf. & & grass & A & 1 & 1 & 0 & 0 & 1 & 0 & 0 \\
\hline 100 & Schismus barbatus (L.) Thell. & & grass & $\mathrm{A}$ & 0 & 0 & 0 & 0 & 0 & 1 & 0 \\
\hline 101 & Setaria verticillata (L.) P.Beauv. & & grass & A & 1 & 1 & 1 & 1 & 0 & 0 & 0 \\
\hline 102 & Setaria viridis (L.) P.Beauv. & & grass & $\mathrm{A}$ & 1 & 1 & 1 & 1 & 0 & 0 & 0 \\
\hline 103 & Stipa capensis Thunb. & & grass & A & 0 & 0 & 0 & 0 & 0 & 1 & 0 \\
\hline 104 & Triticum aestivum L. & & grass & A & 0 & 0 & 0 & 1 & 0 & 0 & 0 \\
\hline 105 & Triticum durum Desf. & & grass & A & 0 & 0 & 0 & 1 & 0 & 0 & 0 \\
\hline & Polygonaceae & $\mathbf{D}$ & & & & & & & & & \\
\hline 106 & Polygonum aviculare $\mathrm{L}$. & & herb & A & 0 & 0 & 1 & 1 & 1 & 0 & 1 \\
\hline 107 & Rumex dentatus $\mathrm{L}$. & & herb & A & 1 & 1 & 1 & 0 & 1 & 0 & 0 \\
\hline 108 & Rumex vesicarius $\mathrm{L}$. & & herb & A & 0 & 0 & 0 & 0 & 0 & 1 & 0 \\
\hline & Solanaceae & D & & & & & & & & & \\
\hline 109 & Hyoscyamus muticus L. & & herb & $\mathrm{P}$ & 0 & 0 & 0 & 1 & 0 & 1 & 0 \\
\hline 110 & Lycopersicon esculentum Mill. & & herb & $\mathrm{A}$ & 1 & 0 & 0 & 1 & 0 & 0 & 0 \\
\hline 111 & Solanum americanum Mill. & & herb & $\mathrm{A}$ & 1 & 1 & 0 & 0 & 1 & 0 & 0 \\
\hline 112 & Withania somnifera (L.) Dunal & & herb & $\mathrm{P}$ & 0 & 0 & 0 & 0 & 1 & 0 & 0 \\
\hline & Zygophyllaceae & D & & & & & & & & & \\
\hline 113 & Fagonia mollis Delile & & herb & $\mathrm{P}$ & 0 & 0 & 0 & 0 & 0 & 1 & 0 \\
\hline 114 & Tribulus terrestris $\mathrm{L}$. & & herb & A & 0 & 0 & 0 & 1 & 1 & 1 & 0 \\
\hline 115 & Zygophyllum album L.f. & & shrub & $\mathrm{P}$ & 0 & 0 & 0 & 0 & 1 & 1 & 0 \\
\hline 116 & Zygophyllum coccineum $\mathrm{L}$. & & shrub & $\mathrm{P}$ & 0 & 0 & 0 & 0 & 1 & 1 & 0 \\
\hline 117 & Zygophyllum simplex $\mathrm{L}$. & & herb & $\mathrm{B}$ & 0 & 0 & 0 & 1 & 1 & 1 & 0 \\
\hline
\end{tabular}

Division: ( $\mathbf{D}=$ Dicotyledons, $\mathbf{M}=$ Monocotyledons $)$. Life span: $(\mathbf{A}=$ Annuals, $\mathbf{B}=$ Biennials, $\mathbf{P}=$ Perennials $)$. Data matrix: $(1=$ presence, $0=$ absence $)$. 
Table (3): List of monotypic and polytypic families with their species numbers and ratios recorded along Cairo - Ismailia desert road.

\begin{tabular}{|c|c|c|c|c|}
\hline No. & & No. of Genera & No. of Species & Ratio of Species \\
\hline & MONOTYPIC FAMILIES & & & \\
\hline 1 & Arecaceae & 1 & 1 & 0.76 \\
\hline 2 & Boraginaceae & 1 & 1 & 0.76 \\
\hline 3 & Cleomaceae & 1 & 1 & 0.76 \\
\hline 4 & Cucurbitaceae & 1 & 1 & 0.76 \\
\hline 5 & Ephedraceae & 1 & 1 & 0.76 \\
\hline 6 & Geraniaceae & 1 & 1 & 0.76 \\
\hline 7 & Juncaceae & 1 & 1 & 0.76 \\
\hline 8 & Neuradaceae & 1 & 1 & 0.76 \\
\hline 9 & Oxalidaceae & 1 & 1 & 0.76 \\
\hline 10 & Portulacaceae & 1 & 1 & 0.76 \\
\hline 11 & Primulaceae & 1 & 1 & 0.76 \\
\hline 12 & Resedaceae & 1 & 1 & 0.76 \\
\hline 13 & Tamaricaceae & 1 & 1 & 0.76 \\
\hline 14 & Typhaceae & 1 & 1 & 0.76 \\
\hline \multirow[t]{2}{*}{15} & Urticaeae & 1 & 1 & 0.76 \\
\hline & POLYTYPIC FAMILIES & & & \\
\hline 16 & Poaceae & 21 & 25 & 18.94 \\
\hline 17 & Compositae & 20 & 21 & 15.91 \\
\hline 18 & Brassicaceae & 9 & 10 & 7.58 \\
\hline 19 & Chenopodiaceae & 7 & 10 & 7.58 \\
\hline 20 & Leguminosae & 7 & 8 & 6.06 \\
\hline 21 & Zygophyllaceae & 4 & 5 & 3.79 \\
\hline 22 & Euphorbiaceae & 4 & 5 & 3.79 \\
\hline 23 & Aizoaceae & 4 & 4 & 3.03 \\
\hline 24 & Amaranthaceae & 3 & 4 & 3.03 \\
\hline 25 & Convolvulaceae & 2 & 4 & 3.03 \\
\hline 26 & Solanaceae & 4 & 4 & 3.03 \\
\hline 27 & Apiaceae & 2 & 3 & 2.27 \\
\hline 28 & Malvaceae & 3 & 3 & 2.27 \\
\hline 29 & Polygonaceae & 2 & 3 & 2.27 \\
\hline 30 & Apocynaceae & 2 & 2 & 1.52 \\
\hline 31 & Caryophyllaceae & 2 & 2 & 1.52 \\
\hline 32 & Cyperaceae & 1 & 2 & 1.52 \\
\hline \multirow[t]{2}{*}{33} & Plantaginaceae & 1 & 2 & 1.52 \\
\hline & Total & 112 & 132 & 100 \\
\hline
\end{tabular}


Table (4): Proximity matrix showed similarity value of monotypic families recorded among the studied habitats along Cairo - Ismailia desert road.

\begin{tabular}{|l|c|c|c|c|c|c|c|}
\hline \multirow{2}{*}{ Habitats } & \multicolumn{7}{|c|}{ Proximity Matrix } \\
\cline { 2 - 8 } & Field crops & Orchards & Gardens & Roadsides & Wastelands & Deserts & Salt marshes \\
\hline Field crops & 1.000 & & & & & & \\
\hline Orchards & 0.833 & 1.000 & & & & & \\
\hline Gardens & 0.600 & 0.500 & 1.000 & & & & \\
\hline Roadsides & 0.333 & 0.286 & 0.500 & 1.000 & & & \\
\hline Wastelands & 0.167 & 0.143 & 0.250 & 0.667 & 1.000 & & \\
\hline Deserts & 0.077 & 0.154 & 0.091 & 0.200 & 0.222 & 1.000 & \\
\hline Salt marshes & $\mathbf{0 . 0 0 0}$ & $\mathbf{0 . 0 0 0}$ & $\mathbf{0 . 0 0 0}$ & 0.200 & 0.250 & 0.091 & 1.000 \\
\hline
\end{tabular}

Table (5): Proximity matrix showed similarity value of polytypic families recorded among the studied habitats along Cairo - Ismailia desert road.

\begin{tabular}{|l|r|r|r|r|r|r|r|}
\hline \multirow{2}{*}{ Habitats } & \multicolumn{7}{|c|}{ Proximity Matrix } \\
\cline { 2 - 9 } & Field crops & Orchards & Gardens & Roadsides & Wastelands & Deserts & Salt marshes \\
\hline Field crops & 1.000 & & & & & & \\
\hline Orchards & 0.607 & 1.000 & & & & & \\
\hline Gardens & 0.469 & 0.463 & 1.000 & & & & \\
\hline Roadsides & 0.324 & 0.361 & 0.469 & 1.000 & & & \\
\hline Wastelands & 0.225 & 0.300 & 0.243 & 0.243 & 1.000 & & \\
\hline Deserts & 0.022 & 0.100 & 0.080 & 0.173 & 0.143 & 1.000 & \\
\hline Salt marshes & $\mathbf{0 . 0 1 9}$ & $\mathbf{0 . 0 5 5}$ & $\mathbf{0 . 0 3 8}$ & 0.038 & 0.119 & 0.058 & 1.000 \\
\hline
\end{tabular}

On the other hand, the lowest ratio (1.9\%) was recorded between field crops and salt marshes habitats. On the whole, the same degree of similarity ratio $24.3 \%$ was noticed between (gardens and wastelands) and (roadsides and wastelands).

3.3. Cluster analysis of monotypic and polytypic families

Based on measured values, the cluster analysis of monotypic and polytypic families classified the studied habitats into five groups namely (G1, G2, G3, G4 and G5). The dendrograms (Figs. $2 \& 3$ ) and (Table 6) of monotypic and polytypic families contained the same habitats at the following groups: G1, G4 and G5. Group 1 included field crops and orchards. Group 4 contained desert habitat and group 5 included salt marshes habitat. On the other hand, groups 2 and 3 contained different habitats at monotypic and polytypic families. Group 2 contained gardens habitat at monotypic families, while; it contained gardens and roadsides habitats at polytypic ones. In addition, group 3 included roadsides and wastelands habitat in monotypic families, while, it contained wastelands at polytypic ones.

3.4. Habit ratios of monotypic and polytypic families

The spectrum of habit ratios (Fig. 4), showed that herb species had the highest contributions $66 \%$ in monotypic followed by $62 \%$ at polytypic families. Shrubs and trees were recorded at monotypic and polytypic families. They 


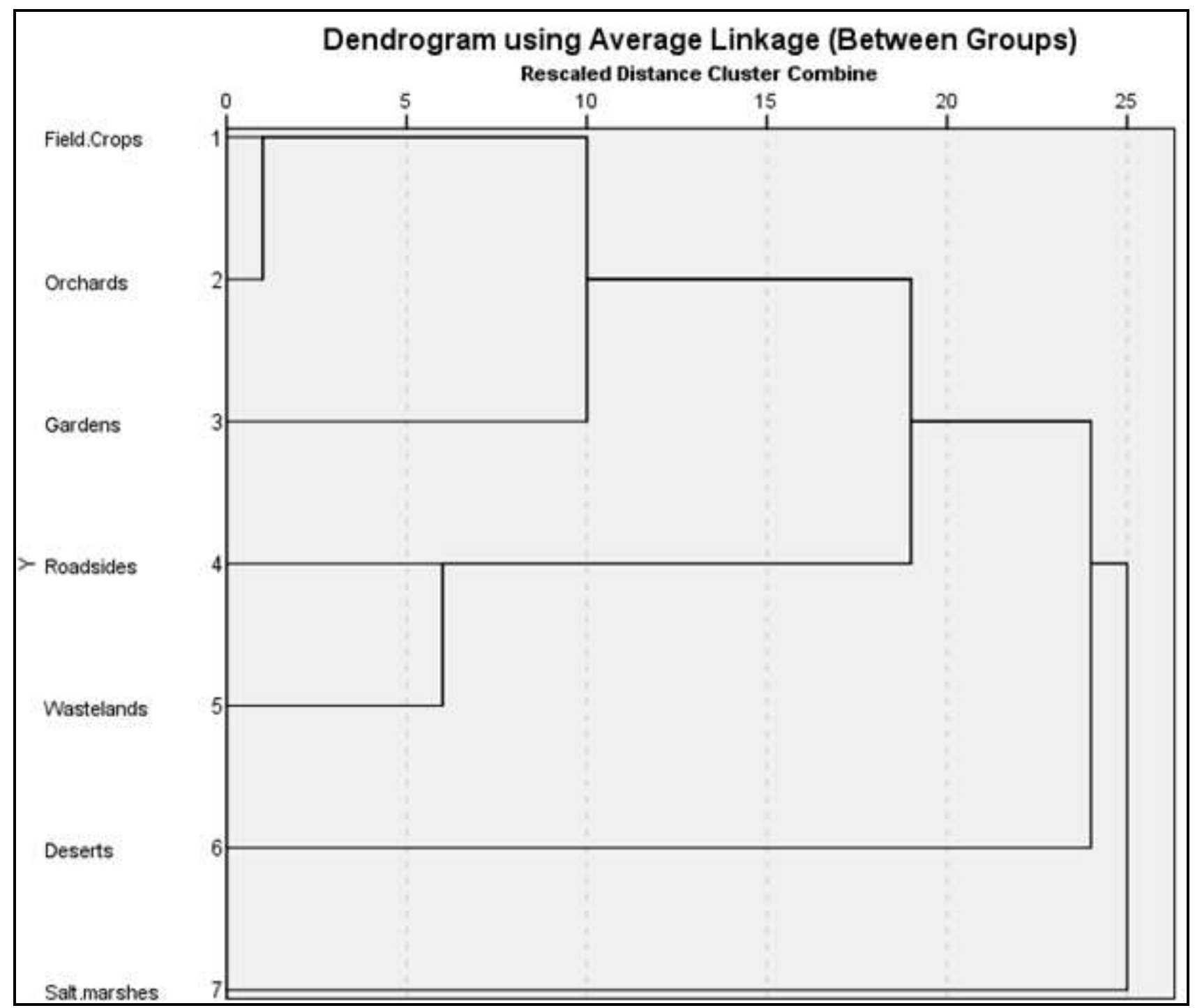

Fig. (2): Dendrogram showed similarity value of monotypic families recorded among the studied habitats along Cairo-Ismailia desert road.

represented by $20 \%$ and $7 \%$ at monotypic families; while there were $11 \%$ and $1 \%$ in polytypic. Rushes (Juncus bufonius L.) were recorded in monotypic families and represented by $7 \%$. On the other hand, grasses, subshrubs and trees were recorded in polytypic families only. They represented by $21 \%, 3 \%$ and $2 \%$, respectively. The low number of shrubs and trees in the current study related to the high intensity of disturbance due to agricultural activities along Cairo-Ismailia desert road; this fact was also reported by Kim et al. (2002), Abd El-Ghani et al. (2013) and Amer et al. (2015). This indicated that the floristic structure of the studied area was affected by human impact (Shaltout and El-Fahar, 1991; Abd El-Ghani et al., 2011 and Amer et al., 2015).
3.5. Life span ratios of monotypic and polytypic families

The spectrums of life span ratios of the studied habitats (Fig. 4) revealed that the perennial species of monotypic families dominated the vegetation diversity among the studied habitats. They represented 53\%, followed by annual herbs $47 \%$. On the other hand, the annual species of monotypic families dominated the vegetation among the studied habitats. They represented $65 \%$, followed by perennials $31 \%$. Moreover, biennual species of polytypic families represented $4 \%$ and not recorded at the monotypic ones (Fig.4). Shaltout and Sharaf El-Din (1988) reported that the flourishing of annuals species at different habitats is related to their great plasticity under 


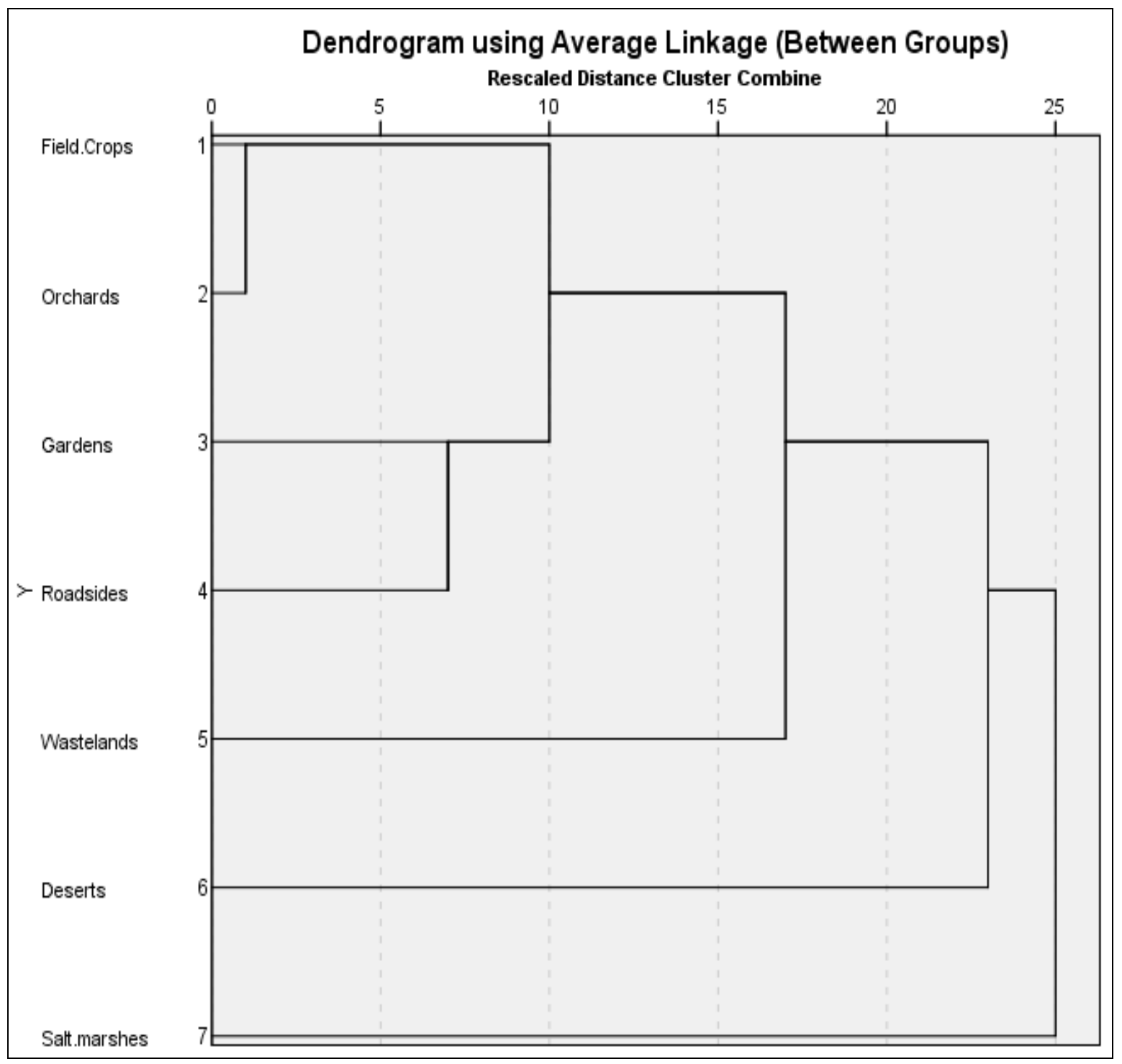

Fig. (3): Dendrogram showed similarity value of polytypic families recorded among the studied habitats along Cairo - Ismailia desert road.

Table (6): Grouping of monotypic and polytypic families among the studied habitats along Cairo- Ismailia desert road.

\begin{tabular}{|l|l|l|}
\hline Group numbers & Monotypic families & Polytypic families \\
\hline G1 & Field crops and Orchards & Field crops and Orchards \\
\hline G2 & (Gardens) & (Gardens and Roadsides) \\
\hline G3 & (Roadsides and wastelands) & (Wastelands) \\
\hline G4 & Deserts & Deserts \\
\hline G5 & Salt marshes & Salt marshes \\
\hline
\end{tabular}

different situations. Abd El-Ghani et al. (2013) reported the short life span ratios of annual species lead to the frequent occurrence during the favorable seasons which supports the present investigation. These explanations are supported by the present investigation based on the recorded species among different habitats.
3.6. Angiospermae and Gymnospermae ratios of monotypic and polytypic families

The study recorded the presence of 33 families included 15 monotypic and 18 polytypic families. The monotypic families were 11 dicotyledons (73\%), 3 monocotyledons (20\%) and 1 chlamydospermae (7\%) families. The 
polytypic families contained 15 dicotyledons (83\%) and 3 monocotyledons (17\%) families (Fig. 4). Angiospermae (dicotyledons and monocotyledons families) constituted the highest ratios of distribution at monotypic and polytypic families. On the other hand, Gymnospermae included chlamydospermae (Ephedra alata Decne.) was recorded only at monotypic families (Fig. 4).

\subsection{Habitat ratios of monotypic and polytypic families}

It was obvious that the monotypic and polytypic families constituted the main bulk of
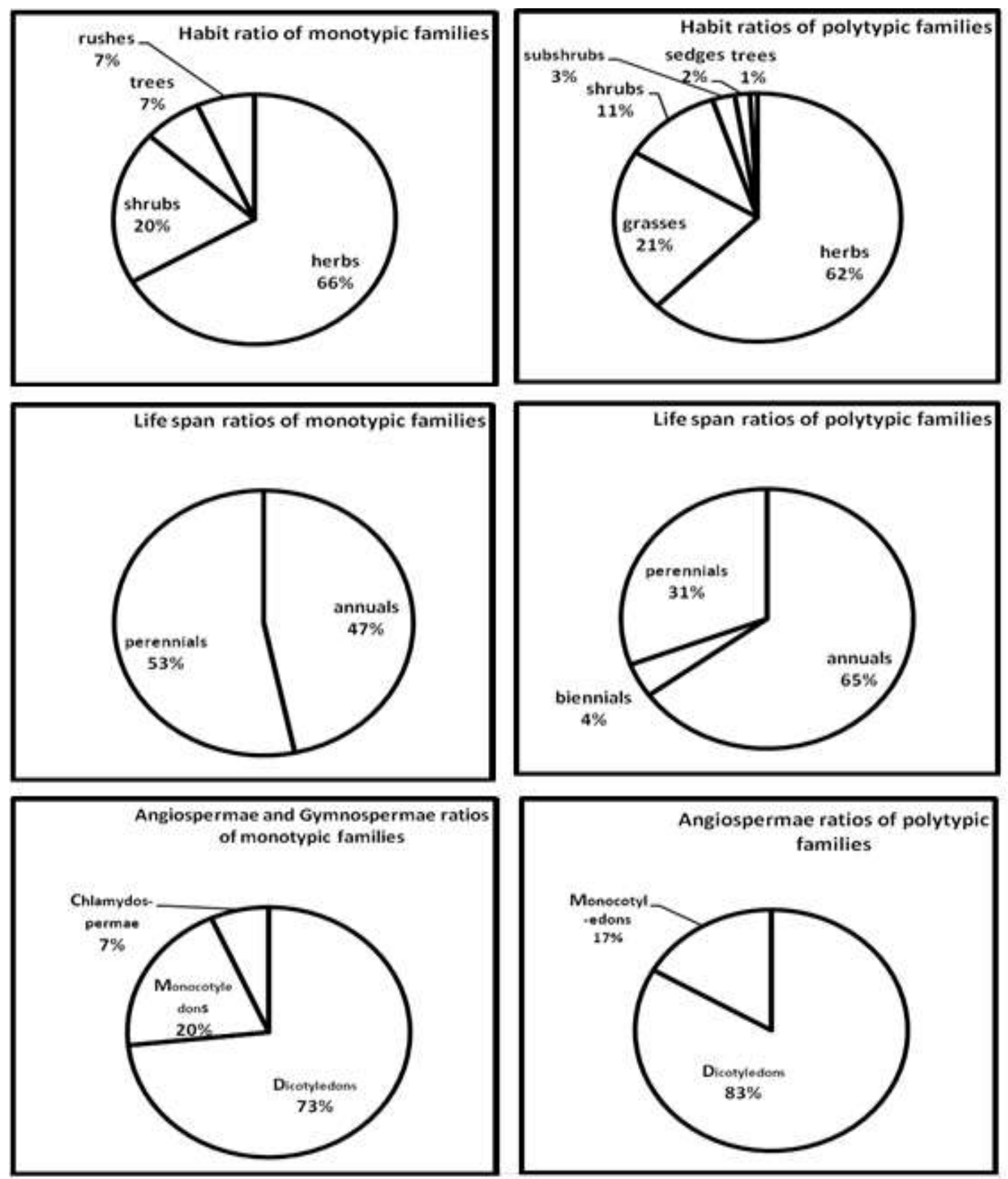

Fig. (4): Spectrum showed ratios of habit, life span and Angiospermae and Gymnospermae of monotypic and polytypic families studied along Cairo-Ismailia desert road. 


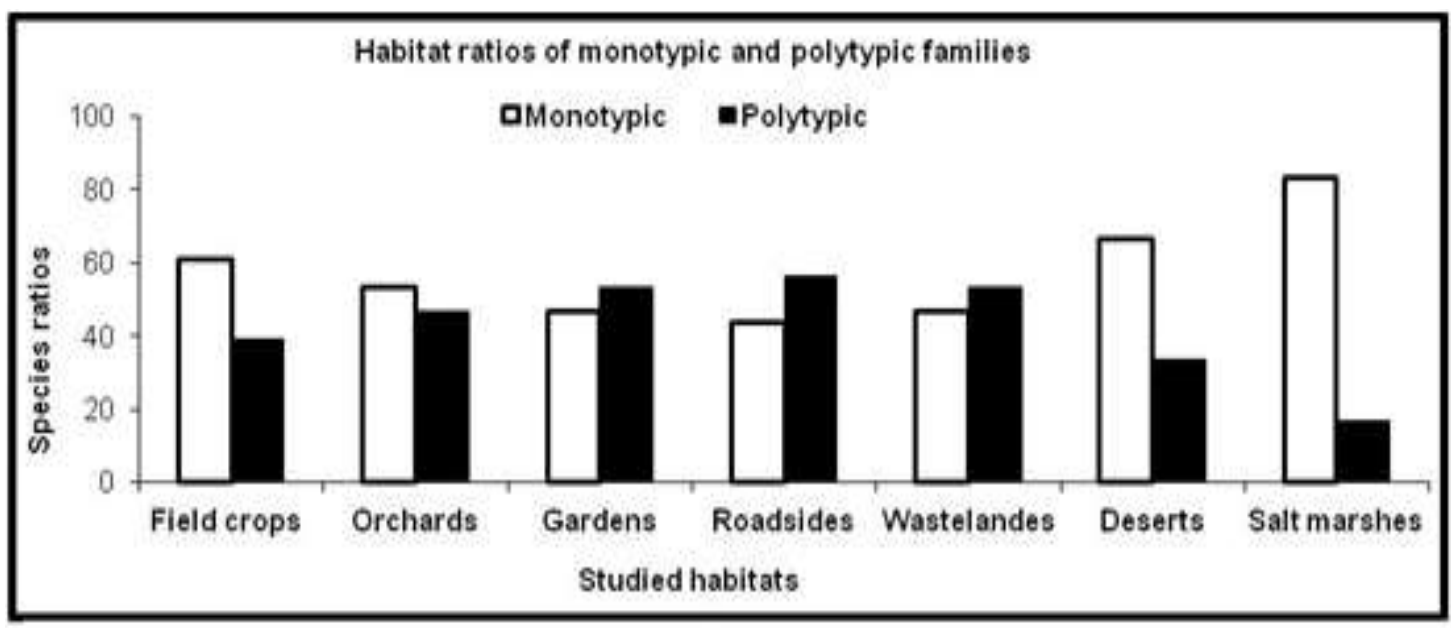

Fig. (5): Histogram showed the habitat ratios of monotypic and polytypic families along Cairo-Ismailia desert road.

\section{Conclusions}

In this study, the analysis of vegetation diversity concluded that the largest distributed polytypic families were Poaceae followed by Compositae then Chenopodiaceae and Brassicaceae. In addition 15 families were monotypic. Herbs dominated at monotypic and polytypic families. The dominant life span species were annuals at polytypic habitats followed by perennial species at monotypic ones. The cluster analysis of monotypic and polytypic families divided the studied habitats into five groups. Moreover, field crops and orchard habitats showed the highest degree of similarity ratios at both monotypic and polytypic families. On the other hand, there was no similarity between species of salt marshes habitat and species of field crops, orchards and gardens.

\section{REFERENCES}

Abd El-Ghani M. M. (1998). Environmental correlates of species distribution in arid desert ecosystems of eastern Egypt. J. Arid Environ., 38 (2): 297-313.

Abd El-Ghani M. M. and Fawzy A. M. (2006). Plant diversity around springs and wells in five oases of the Western Desert, Egypt. Int'l., J. Agric. Biol., 8 (2): 249255.

Abd El-Ghani M. M., Bornkamm R., El-Sawaf N. and Turkey H. (2011). Plant species distribution and spatial habitat heterogeneity in the landscape of urbanizing desert ecosystem of Egypt. Urban Ecosyst., 14 (4): 585-616.
Abd El-Ghani M. M., Soliman A. T., Hamdy R. and Bennoba E. (2013). Weed flora in the reclaimed lands along the northern sector of the Nile Valley in Egypt. Turk. J. Bot., 37 (3): 464-488.

Amer W., Soliman A. and Hassan W. (2015). Floristic composition of Nile islands in Middle Egypt with special reference to the species migration route. J. Amer. Sci., 11 (6): 14-23.

Angiosperm Phylogeny Group III. (2009). an update of the Angiosperm Phylogeny Group classification for the orders and families of flowering plants: APG III". Bot. J. the Linn Soc., 161 (2): 105-121.

Azer S. A. (2013). Study on the plant diversity in Saqqara region, Giza, Egypt. Egypt J. Biotech., 45 (2): 1-17.

Boulos L. (1995). Flora of Egypt Checklist. Al Hadara Publishing, Cairo, Egypt. pp.1283.

Boulos L. (1999). Flora of Egypt. vol. 1 (Azollaceae-Oxalidaceae). Al-Hadara Publishing, Cairo, Egypt. pp.1-419.

Boulos L. (2000). Flora of Egypt. vol. 2 (Geraniaceae-Boraginaceae). Al-Hadara Publishing, Cairo, Egypt. pp.1-352.

Boulos L. (2002). Flora of Egypt. vol. 3 (Verbinaceae - Compositae). Al-Hadara Publishing, Cairo, Egypt. pp.1-373.

Boulos L. (2005). Flora of Egypt. vol. 4 (Monocotyledons: Alismataceae Orchidaceae). Al Hadara Publishing, Cairo, Egypt. pp.1-617.

Boulos L. (2008). Flora and vegetation of the deserts of Egypt. Flora Mediter. 
18: $341-359$.

Boulos L. (2009). Flora of Egypt checklist (Revised annotated edition). Al Hadara Publishing Cairo Egypt. pp.1-410.

Chase M. W. and Reveal J. L. (2009). A phylogenetic classification of the land plants to accompany APG III". Bot. J. Linn. Soc., 161 (2): 122-127.

El-Hadidi N. and Fayed A. (1994/95). Materials for excursion flora of Egypt. Cairo University Herbarium. Täeckholmia, 15, pp.1-233.

Fawzi N. M., Abdelmaksoud H. S. and Azer S. A. (2017). Floristic composition of Dahab Island, at Nile region, Egypt. Curr. Sci. Int'1., 6 (4); 729-745.

Hamed S. T., Sheded M. G. and Owis M. (2012). Floristic composition of some riverian islands at Qena governorateEgypt. $2^{\text {nd }}$ International conference at Minia University, Egypt J. Bot., pp. 299322.

Haston E., Richardson J. E., Stevens P. F., Chase M. W. and Harris D. J. (2009). The Linear Angiosperm Phylogeny Group (LAPG) III: a linear sequence of the families in APG III. Bot. J. Lin Soc., 161 (2): 128-131.

Kassas M. (1953a). Habitats and plant communities in the Egyptian deserts: I. Introduction. J. Ecol., 40 (2): 342-351.

Kassas M. (1953b). Habitats and plant communities in the Egyptian deserts: II. The features of a desert community. J.Ecol., 41(2): 248-256.

Kassas M. (1954). Habitats and plant communities in the Egyptian deserts: III. The wadi bed. J. Ecol., 41(2): 424-441.

Kassas M. and El-Abyad M. S. (1962). On the Phytosociology of the desert vegetation of Egypt. Ann. of Arid Zone, 1(1): 5483.

Kassas M. and Girgis W. A. (1964). Habitats and plant communities in the Egyptian deserts. The limestone plateau. J. Ecol., 52 (1):107-119.

Kassas M. and Girgis W. A. (1965). Habitat and plant communities in the Egyptian desert. VI. The units of a desert ecosystem. J. Ecol., 53 (3): 715-728.

Kim Y. M., Zerbe S. and Kowarik I. (2002). Human impact on flora and habitats in Korean rural settlements. J. Preslia, 74 (4): 409-419.
Mashaly I. A., El-Habashy I. E., El-Halawany E. F. and Botany G. O. (2009). Habitat and plant communities in the Nile delta of Egypt. II. Irrigation and drainage canal bank habitat. Pak. J. Biol. Sci., 12 (12): 885-895.

Salama F. M. and Fayed A. A. (1989). Phytosociological studies along the IdfuMarsa Alam road. Feddes Repert., 100 (3-4): 191-195.

Salama F. M. and Fayed A. A. (1990). Phytosociological study on the deltaic part and the principal channel of wadi Qena, Egypt. Feddes Repert., 101 (12): 89-95.

Salama F. M. and El-Naggar S. M. (1991). Phytosociology of wadi system west of Qusseir province. Feddes Repert., 102 (5-6): 453-468.

Salama F., Abd El-Ghani M. and El-Tayeh N. (2013). Vegetation and soil relationships in the inland wadi ecosystem of central Eastern Desert, Egypt. Turk. J. Bot., 37 (3): 489-498.

Shaheen A. M. (2002). Weed diversity of newly farmed land on the southern border of Egypt (Eastern and Eastern shores of Lake Nasser). Pak. J. Biol. Sci., 5 (4): 602-608.

Shaltout K. H. and Sharaf El-Din A. (1988). Habitat types and plant communities along a transect in the Nile Delta region. Feddes Repert., 99 (3-4): 153-162.

Shaltout K. H. and El-Fahar R. (1991). Diversity and phenology of weed communities in the Nile Delta region. J. Veget. Sci., 2 (3): 385-390.

SPSS (2013). IBM SPSS Statistics for Windows, Version 22.0. Armonk, NY: IBM Corp., USA. pp.1-250.

Täckholm V. (1974). Students' Flora of Egypt. $2^{\text {nd }}$ ed., Cairo University. Pub by Cooperative Printing Company Beirut. Lebanon pp. 42-790.

Zahran M. A. and Willis A. J. (2009). The vegetation of Egypt. $2^{\text {nd }}$ ed. Springer Publication. Cairo, Egypt pp.1-427.

Zahran M. A. and El-Amier Y. A. (2014). Ecology and establishment of fiber producing taxa naturally growing in the Egyptian deserts. Egypt. J. Basic and Appl. Sci., 1(3-4): 144-150. 


\section{دراسة على تنوع الكساء الخضرى خلال طريث القاهرة ـ الاسماعيلية الصحراوى، مصر صفوث أمين عازر \\ قسم بحوث الفلورة وتصنيف النباتات ـ معهد بحوث البساتين - مركز البحوث الزر اعية ـ الجيزة ـ مصر \\ ملخص}

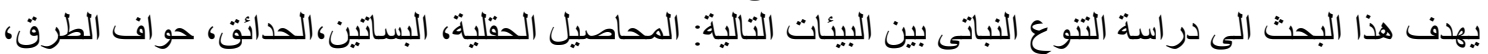

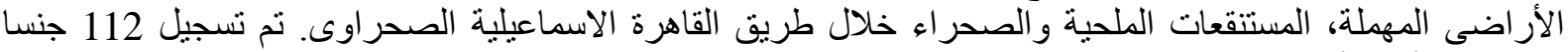

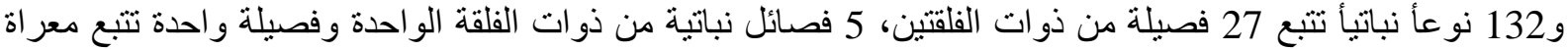

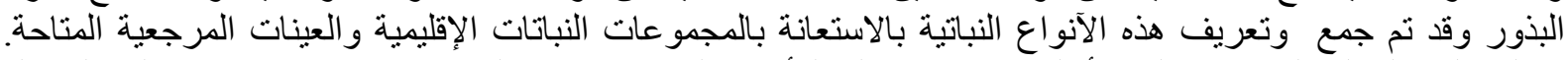

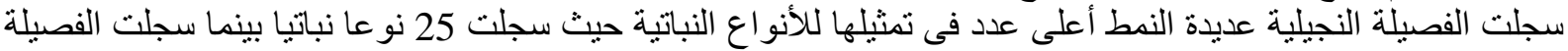

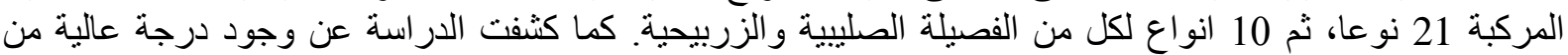

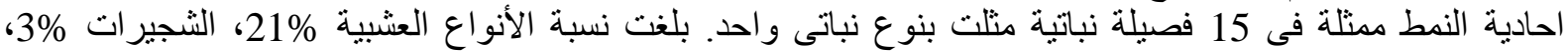
و الاحر اش 2\% فى الفصائل عديدة النمط فقط. مثلت الحوليات باعلى نسبة الانواع ثنائية الحول بنسبة 4\% في الفصائل وحيدة النمط فقط. أظهرت التحليلات العنقودية درجة كبيرة من التشابه بين

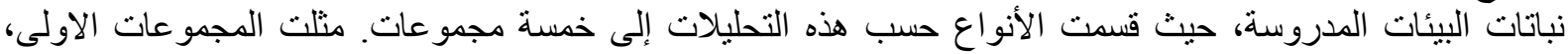

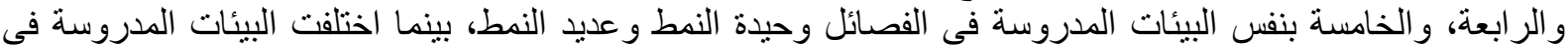
المجمو عات الثانية والثالتة فى الفصائل وحيدة النمط وعديدة النمط. لوحظ أن نباتات المحاصيل الحقلية و البساتين لديهما أعلى نسبة من التشابه قيمتة 83\% في الفصائل وحيدة النمط و $10.7 \%$ فى الفصائل عديدة النمط. في حين لم بكن هنالك اي الى درجة من التشابه بين نباتات بيئة المستنقعات الملحية و البيئات التالية: المحاصيل الحقلية، البساتين و الحدائق فى الفصائل وحيدة النمط فقط مجن.

المجلة العلمية لكلية الزراعة -جامعة القاهرة ـ المجلا (69) العدد الاول (يناير 2018 ) : 98-85. 